\title{
Dominik Noll
}

On the theory of $B$ - and $B_{r}$-spaces in general topology

Czechoslovak Mathematical Journal, Vol. 39 (1989), No. 4, 589-594

Persistent URL: http://dml.cz/dmlcz/102332

\section{Terms of use:}

(C) Institute of Mathematics AS CR, 1989

Institute of Mathematics of the Czech Academy of Sciences provides access to digitized documents strictly for personal use. Each copy of any part of this document must contain these Terms of use.

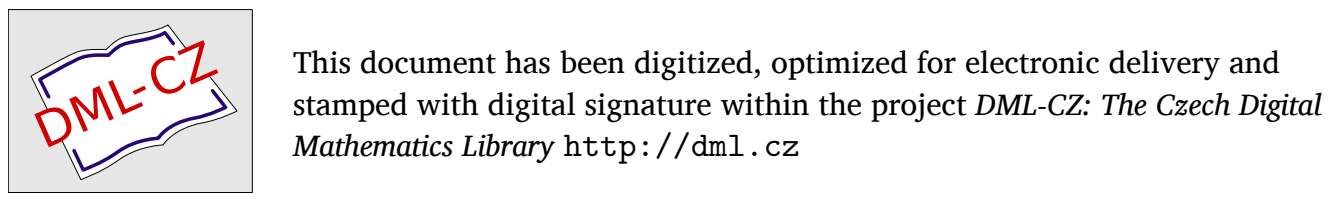




\title{
ON THE THEORY OF $B$ - AND $B_{r}$-SPACES IN GENERAL TOPOLOGY
}

\author{
D. NoLL, Stuttgart
}

(Received August 5, 1986)

1. $B$ - and $B_{r}$-spaces. A $T_{2}$ topological space $E$ is called a $B_{r}$-space (B-space) if every continuous, nearly open bijection (surjection) $f$ from $E$ onto an arbitrary $T_{2}$ space $F$ is open. Here $f: E \rightarrow F$ is called nearly open if for every $x \in E$ and every neighbourhood $U$ of $x$ the set $\operatorname{cl}(f(U))$ is a neighbourhood of $f(x)$.

The notions of $B$ - and $B_{r}$-spaces in the above sense have first been used by $T$. Husain in the categories of locally convex vector spaces $\left(\left[\mathrm{Hu}_{1}\right]\right)$ and topological groups $\left(\left[\mathrm{Hu}_{2}\right]\right)$. They have been chosen in reminiscence of V. Pták's open mapping theorems $([\mathrm{P}],[\mathrm{Kö}])$. We have adopted Husain's definition for the topological case. References concerning the classical theory of $B$ - and $B_{r}$-spaces and groups are $[\mathrm{P}],[\mathrm{Kö}],[\mathrm{AEK}],\left[\mathrm{Hu}_{i}\right],\left[\mathrm{Ba}_{i}\right],[\mathrm{Pe}],[\mathrm{Gr}],[\mathrm{Su}]$, etc. In a purely topological context, $B_{r}$-spaces have been considered in $[\mathrm{We}],[\mathrm{BP}]$, although the term ' $B_{\boldsymbol{r}}$-space' has not been used there. Further references are [Wi], $[\mathrm{St}],\left[\mathrm{N}_{i}\right]$.

Every $T_{2}$ locally compact space is a $B$-space and every $B$-space is a $B_{r}$-space. In [We], Weston proved that every completely metrizable space is a $B_{r}$-space. In [BP] this has been generalized to Čech complete spaces. In $\left[N_{1}\right]$ we have further generalized this to obtain.

Proposition 1. Every $T_{2}$ semi-regular topological space $E$ containing a dense Čech complete subspace is a $B_{r}$-space. In particular, this is true for monotonically Čech complete spaces.

In $\left[\mathrm{N}_{1}\right]$ we have given a direct proof. Proposition 1 may also be deduced from Byczkowski and Pols' result $[\mathrm{BP}]$ if we use the following

Lemma. Let $E$ be a $T_{2}$ semi-regular space and let $F$ be a $T_{2}$ space. Let $f: E \rightarrow F$ be a continuous, nearly open bijection and suppose there exists a dense subset $D$ of $E$ such that $f \mid D: D \rightarrow f(D)$ is open. Then $f$ is open.

Proof. Let $x \in E$ and a neighbourhood $U$ of $x$ be fixed. Choose a regular-open neighbourhood $V$ of $x$ contained in $U$. We prove int $\operatorname{cl}(f(V)) \subset f(U)$. Let $z \in$ $\in$ int $\operatorname{cl}(f(V)), z=f(y)$. Let $W$ be a neighbourhood of $y$ with $f(W) \subset \operatorname{int} \operatorname{cl}(f(V))$. It is sufficient to prove $W \subset \bar{V}$. So let $w \in W$ and let $O$ be a regular-open neighbourhood of $w$ contained in $W$. Proving that $O \cap V \neq \emptyset$ remains. 
Since $O, V$ are regular-open in $E, O \cap D, V \cap D$ are regular-open in $D$, hence $f(O \cap D), f(V \cap D)$ are regular-open in $f(D)$. But note that int $\operatorname{cl}(f(O)) \cap f(D)$ and int $\mathrm{cl}(f(V)) \cap f(D)$ are as well regular-open in $f(D)$ and this implies int $\operatorname{cl}(f(O)) \cap$ $\cap f(D)=f(O) \cap f(D)$, int $\operatorname{cl}(f(V)) \cap f(D)=f(V) \cap f(D)$. Since $O \subset W$ implies int $\mathrm{cl}(f(O)) \subset$ int $\operatorname{cl}(f(V))$ we obtain the desired result $O \cap V \neq \emptyset$.

In $\left[\mathrm{N}_{3}\right]$ we have investigated an interesting class of $B$-spaces.

Proposition 2. Every Lindelöf $P$-space is a B-space.

Using the lemma above, one may obtain the following result. Here 'locally Lindelöf' means that every point has a base of neighbourhoods consisting of Lindelöf subspaces.

Proposition 3. Every $T_{2}$ semi-regular locally Lindelöf space E containing a dense set of $P$-points is a $B_{r}$-space.

Proof. Let $f: E \rightarrow F$ be a continuous, nearly open bijection onto the $T_{2}$ space $F$. We may assume that $F$ is semi-regular. Let $D$ denote the set of $P$-points in $E$. We prove that $f \mid D: D \rightarrow f(D)$ is open. First note that every point of $f(D)$ is a $P$-point in $F$. Indeed, let $G_{n}, n=1,2, \ldots$ be open sets containing $y=f(x), x \in D$. Choose open sets $V_{n}, n=1,2, \ldots$ in $E$ having $x \in V_{n}$, int $\operatorname{cl}\left(f\left(V_{n}\right)\right) \subset G_{n}$. Then $V=\bigcap_{n} V_{n}$ is a neighbourhood of $x$ having int $\operatorname{cl}(f(V)) \subset G_{n}, n=1,2, \ldots$.

Let $x \in D$ and a Lindelöf neighbourhood $U$ of $x$ be fixed. We claim that $\operatorname{cl}(f(U)) \cap$ $\cap f(D)=f(U) \cap f(D)$. Assume the contrary and let $z \in \operatorname{cl}(f(U)) \backslash f(U), z=f(y)$, $y \in D$. Let $\Phi$ denote the filter of neighbourhoods of $z$, then $\{f(U) \backslash \bar{O}: O \in \Phi\}$ is an open cover of $f(U)$, hence there exist $O_{n} \in \Phi, n=1,2, \ldots$ having $f(U)=U_{n} f(U) \backslash O_{n}$, a contradiction since we have $\bigcap_{n} O_{n} \in \Phi$.

It follows from our lemma that every $T_{2}$ semi-regular space $E$ containing a dense $B_{r}$-subspace is itself a $B_{r}$-space. The corresponding result for $B$-spaces is not valid. In $\S 7$ we shall present an example of a completely regular space $E$ containing a dense Lindelöi $P$-subspace which is not a $B$-space.

In $\left[\mathrm{N}_{2}\right]$ we have investigated another interesting class of $B_{r}$-spaces. Let $S$ be a cofinal subset of $\omega_{1}$. Let $S^{*}$ denote the set of $f \in \omega_{1}^{\omega}$ having $f^{*}=\sup \{f(n): n<\omega\} \in$ $\in S$. Give $\omega_{1}$ the discrete topology and let $\omega_{1}^{\omega}$ and $S^{*}$ have the product topology. Recall that $S$ is called stationary if it intersects every closed cofinal subset of $\omega_{1}$. We have the following

Proposition 4. ([N $\left.\mathrm{N}_{2}\right],[\mathrm{FK}]$ for $\left.(1) \Leftrightarrow(2)\right)$. Let $S \subset \omega_{1}$ be cofinal. Then the following statements are equivalent:

(1) $S$ is stationary;

(2) $S^{*}$ is a Baire space;

(3) $S^{*}$ is a $B_{r}$-space.

This provides examples of metrizable $B_{r}$-spaces which do not contain any dense completely metrizable subspace, since clearly $S^{*}$ contains a dense completely metrizable subspace if and only if $S$ contains a closed cofinal subset. 
2. Order interpretation. We introduce an order relation $\leqq$ on the set of all $T_{2}$ topologies on a fixed set $E$ by postulating that $\tau_{1} \leqq \tau_{2}$ is satisfied if and only if id: $\left(E, \tau_{2}\right) \rightarrow\left(E, \tau_{1}\right)$ is continuous and nearly open. Then $(E, \tau)$ is a $B_{r}$-space if and only if $\tau$ is minimal among $T_{2}$ topologies on $E$. Dually one may consider the $\leqq$ maximal topologies. It turns out that these can be internally characterized as follows.

Proposition 5. $\tau$ is maximal with respect to $\leqq$ if and only if every dense subset of $(E, \tau)$ is open.

Open problem. Obtain an internal characterization of $\leqq$ minimal (i.e. $B_{\mathbf{r}_{-}}$) topologies.

Using the Kuratowski/Zorn lemma one easily proves that given any $T_{2}$ topology $\tau$ on $E$, there exists $a \leqq$ maximal topology $\tau_{0}$ having $\tau \subset \tau_{0}$.

Open problem. Does a corresponding result hold for $\leqq$ minimality?

3. Category. Since $T_{2}$ minimal ( $=H$ minimal) topological spaces are clearly $B_{r}$-spaces, it follows from a result of Herrlich ([He]) that a $B_{r}$-space need not be a Baire space in general. One may ask, however, for a first category $B_{r}$-space which is completely regular. In $\left[\mathrm{N}_{3}\right]$ we have provided an example of this type constructing a first category Lindelöf $P$-space. On the other hand, all metrizable $B_{r}$-spaces known up to now are Baire spaces. In $\left[\mathrm{N}_{3}\right]$ we have obtained the following

Theorem 1. Every strongly zero-dimensional metrizable $B_{\boldsymbol{r}}$-space is Baire.

Open problem. Is it true that every metrizable $B_{r}$-space is a Baire space?

Note that theorem 1 may be used to prove that every suborderable metrizable $B_{r}$-space is a Baire space. Another partial positive answer is obtained for metrizable topological groups in view of the following

Proposition 6. ([ $\left[\mathrm{N}_{2}\right]$ ) Every topological group which is a $B_{r}$-space (in the topological sense) is complete with respect to its two-sided uniformity.

4. Products. The situation in the classical categories (see [Kö], $[\mathrm{Gr}]$ ) suggests that the product of even two $B_{r}$-spaces need not be a $B_{r}$-space. In $\left[\mathrm{N}_{2}\right]$ we have obtained the expected counterexamples.

Proposition 7. Let $S, T \subset \omega_{1}$ be stationary sets. Then the following are equivalent:

(1) $S \cap T$ is stationary;

(2) $S^{*} \times T^{*}$ is a $B_{r}$-space.

Clearly this provides the desired counterexamples for we may choose disjoint stationary subsets $S, T$ of $\omega_{1}$, then $S^{*}, T^{*}$ are $B_{r}$-spaces, but $S^{*} \times T^{*}$ is nor.

One may ask for a $B_{r}$-space $E$ whose square $E \times E$ is no longer a $B_{r}$-space. Such an example can be obtained from the following construction. 
Proposition 8. Let $F$ be a strongly zero-dimensional metrizable Baire space such that for some $n \geqq 2 F^{n}$ is no longer a Baire space. Suppose that $F$ is a $B_{r}$-space. Then there exists $r, 1 \leqq r \leqq n-1$ such that $E=F^{r}$ is a $B_{r}$-space but $E \times E$ is not.

Proof. The construction is based on theorem 1 and the fact that finite products of strongly zero-dimensional metrizable spaces are strongly zero-dimensional and metrizable. Regard $F \times F$. If this is not a $B_{r}$-space, then $E=F$. Otherwise $F^{2}$ is a Baire space by theorem 1 . Then regard $F^{2} \times F^{2}$. If this is not $B_{r}$, then $E=F^{2}$. Otherwise $F^{4}$ is a Baire space. etc.

In $\left[\mathrm{N}_{3}\right]$ we have obtained a space $F$ as above using an example from [FK].

Though no general positive results concerning products of $B_{r}$-spaces are to be expected, there are positive results in special situations. Namely the classes of $T_{2}$ minimal spaces, Čech complete spaces, Lindelöf $P$-spaces are examples of productive, countably productive, finitely productive classes of $B_{r}$-spaces.

Open problem. Given a $B_{r}$-space $E$ and a compact $T_{2}$ space $K$, must $E \times K$ be a $B_{r}$ space?

5. Closed subspaces. From the situation in the classical categories (concerning the open mapping theory) one would expect that closed subspaces of $B_{r}$-spaces are again $B_{r}$. In fact, the corresponding statements are known to be valid in the categories of locally convex vector spaces $([\mathrm{Kö}])$, linear topological spaces ([AEK]) and Abelian topological groups. In the case of topological groups the answer is not known (see $\left[\mathrm{Ba}_{2}\right],[\mathrm{Gr}]$ ) although there are some positive partial results. In the topological case, the situation seems to be of a completely different nature for we have the

Proposition 9. Every $T_{2}$ semi-regular topological space $E$ is the closed subspace of some $B_{r}$-space $F$.

Proof. Let $F=E \times\{1\} \cup E \times\{2\}$ and define a topology on $F$ by imposing that $\{(x, 1)\}$ is a neighbourhood of $(x, 1)$ for each $x \in E$ and $U(x)$ is a neighbourhood of $(x, 2)$, whenever $x \in E$ and $U$ is a neighbourhood of $x$ in $E$, where $U(x)$ denotes the set $\{(y, i): y \in U \backslash\{x\}, i=1,2\} \cup\{(x, 2)\}$. Then $E \times\{2\}$ is a closed subspace of $F$ homeomorphic with $E$ and $E \times\{1\}$ is an open dense and discrete subspace of $F$. Since $\dot{F}$ is semi-regular by construction, it is a $B_{\boldsymbol{r}}$-space by proposition 1 .

6. Sums of $B_{r}$-spaces. The class of $B_{r}$-spaces behaves very strange with respect to summation. First note that the sum of even two $B_{r}$-spaces need not be a $B_{r}$-space. Indeed, let $S, T$ be disjoint stationary subsets of $\omega_{1}$, then $S^{*}, T^{*}$ are $B_{r}$-spaces but $S^{*}+T^{*}$ is not $B_{r}$ in view of the fact that $S^{*}, T^{*}$ are disjoint dense subspace of $\omega_{1}^{\omega}$ and hence the natural mapping $f: S^{*}+T^{*} \rightarrow \omega_{1}^{\omega}$ is a continuous nearly open bijection onto $f\left(S^{*}+T^{*}\right)$ which is not open.

On the other hand there are certain positive results on sums of $B_{r}$-spaces. 
Proposition 10. $\left(\left[\mathrm{N}_{2}\right]\right)$ Given any $B_{r}$-space $E$, the sum $E+E$ is a $B_{r}$-space.

In $\left[\mathrm{N}_{2}\right]$ we have investigated summation with Čech complete summands and have obtained the following interesting

Theorem 2. Let $E$ be a completely regular $B_{r}$-space. Then the following statements are equivalent:

(1) E is a Baire space;

(2) $E+F$ is a $B_{r}$-space whenever $F$ is Čech complete.

As a consequence of theorem 1 and theorem 2 we deduce that $E+F$ is a $B_{r}$-space if $E$ is a strongly zero-dimensional metrizable $B_{r}$-space and $F$ is Čech complete. On the other hand, if $E$ is a Lindelöf $P$-space of the first category, theorem 2 provides a Čech complete space $F$ such that $E+F$ is no longer a $B_{r}$-space.

Another positive result on sums is the following

Proposition 11. Given a $B_{r}$-space $E$ and $a T_{2}$ locally compact space $L$, the sum $E+L$ is a $B_{r}$-space.

Proof. Let $f: E+L \rightarrow F$ be a continuous, nearly open bijection onto the $T_{2}$ space $F$. Since $f|E: E \rightarrow f(E), f| L: L \rightarrow f(L)$ are as well nearly open, we have $E \simeq f(E), L \simeq f(L)$. It remains to prove that $f(E)$ is closed in $F$. But this follows from the fact that $f(L)$ is open in its $T_{2}$ extension int $\mathrm{cl}(f(L))$ and so is open in $F$.

7. $B$-spaces. It has been an open question for a long time whether there exist $B_{r}$-complete locally convex vector spaces which are not $B$-complete. Finally, an example of this type has been found by Valdivia ([V]). In the category of topological groups the corresponding counterexample was constructed in $[\mathrm{Su}]$. Now in the purely topological case the situation is different. While the class of $B_{r}$-spaces is considerably large, $B$-spaces seem to be of a rather special type. In fact, even completely metrizable spaces need not be $B$-spaces. An example may be found in [BP].

Example. A $T_{2}$ minimal space need not be a $B$-space. Indeed, let $E$ denote the $T_{2}$ minimal space constructed in [He], whose point set is $R_{0} \cup R_{1} \cup R_{2}$, where $R_{0}=$ $=\mathbb{R} \backslash \mathbf{Q} \cap I \times\{0\}, R_{i}=\mathbf{Q} \cap I \times\{i\}, i=1,2$. Define $f: E \rightarrow I$ by $f(x, i)=x$, then $f$ is a continuous, nearly open surjection which is not open.

Concerning sums of $B$-spaces we have the following

Proposition 12. $\left(\left[\mathrm{N}_{3}\right]\right)$. Let E be a completely regular B-space. Then the following statements are equivalent:

(1) $E+L$ is a B-space whenever Lis $T_{2}$ locally compact;

(2) $E+K$ is a B-space whenever $K$ is $T_{2}$ compact;

(3) $E+\beta E$ is a $B$-space;

(4) E is locally compact.

Let $E$ be a non-discrete Lindelöf $P$-space. Then $E$ is a $B$-space but $E+\beta E$ is not 
since $E$ is not locally compact. On the other hand, $E+E$ is clearly a $B$-space since it is Lindelöf $P$. This proves that the lemma from $\S 1$ is not valid for surjective mappings $f$ resp. the class of $B$-spaces is not closed with respect to taking $T_{2}$ extensions.

\section{Bibliography}

[AEK] N. Adasch, B. Ernst, D. Keim: Topological vector spaces LNM 639.

[Ba $\left.{ }_{1}\right] \quad J . W$. Baker: Inversion theorems and compactness. JLMS 47 (1976), $409 \mathrm{ff}$.

$\left[\mathrm{Ba}_{2}\right] \quad J . W$. Baker: On a generalized open mapping theorem. MA 172 (1976), $217 \mathrm{ff}$.

[BP] T. Byczkowski, R. Pol.: On the closed and open mapping theorems. Bull. Acad. Pol. Sci. 24 (1976), 723-726.

[FK] W. G. Fleissner, K. Kunen: Barely Baire spaces. FM CI (1978), 229-240.

[Gr] D. L. Grant: Topological groups which satisfy an open mapping theorem. PJM 68 (1977), 411-423.

[He] $H$. Herrlich: Nicht alle $T_{2}$ minimalen Räume sind von zweiter Kategorie. MZ 91 (1966), 15.

[Hu $\mathrm{Hu}_{1}$ T. Husain: The open mapping and closed graph theorem in topological vector spaces. Braunschweig 1965.

[ $\left.\mathrm{Hu}_{2}\right]$ T. Husain: Introduction to topological groups. Philadelphia 1966.

[Kö] G. Köthe: Topological vector spaces II. New York 1979.

$\left[\mathrm{N}_{1}\right]$ D. Noll: Open mapping theorems in topological spaces. CMJ 35 (110), 1985.

$\left[\mathrm{N}_{2}\right] \quad$ D. Noll: Sums and products of $B_{r}$-spaces. PJM 123, 1, 1986.

$\left[\mathrm{N}_{3}\right] \quad$ D. Noll: Baire category and $B_{r}$-spaces. PAMS 101, $671 \mathrm{ff}, 1987$.

[Pe] B. J. Pettis: On continuity and openness of homomorphisms in topological groups. Ann. of Math. 52 (1957), 293-308.

[P] V. Pták: Completeness and the open mapping theorem. Bull. Soc. Math. France 86, 41-74, 1958.

[St] J. D. Stein: The open mapping theorem for spaces with unique segments. PJM 40 (2), 1972, 459-462.

[Su] L. J. Sulley: A note on $B$ - and $B_{r}$-complete topological Abelian groups. Proc. Camb. Phil. Soc. 66 (1969), 275.

[We] J. D. Weston: On the comparison of topologies. JLMS 32 (1957), 342-354.

[Wi] M. Wilhelm: Criteria for the openness of relations. FM CXIV ('81), $219 \mathrm{ff}$.

Author's address: Universität Stuttgart, Mathematisches Institut B, Pfaffenwaldring, 7000 Stuttgart 80, BRD. 